\title{
Dependence of thermal conductivity of snow on microstructure
}

\author{
P K SATYAWALi ${ }^{1, *}$ and A K Singh ${ }^{2}$ \\ ${ }^{1}$ Snow and Avalanche Study Establishment, Him Parisar Sector 37A, Chandigarh, India. \\ ${ }^{2}$ Defence Institute of Advanced Technology (Deemed University), Girinagar, Pune, India. \\ *e-mail: pramodsatyawali@hotmail.com
}

\begin{abstract}
A geometrical model, including different geometrical shapes influencing thermal conductivity of snow is proposed. The geometrical model has been assumed to comprise of unit cells having solid (ice) inclusion as an aggregation of spherical, cylindrical or cubical shapes with vertical connection, arranged in a cubic packing. From the geometrical model and one-dimensional heat transfer theory, the effective thermal conductivity has been computed. For this purpose, coupled one-dimensional heat transfer equations have been solved for steady-state condition to account for conduction in ice, conduction in air and latent heat transfer due to water vapour sublimation through air. The model demonstrates the dependency of thermal conductivity on density, grain-spacing, grain contact ratio and temperature. Spherical inclusions give highest conductivity while cubical inclusion estimates lowest value for the same density. Thermal conductivity has been found increasing sharply near to the packing density for all three shapes. Empirical model results and results obtained from existing microstructure based models have also been compared with the present model.
\end{abstract}

\section{Introduction}

A review (Sturm et al 1997) of thermal conductivity $\left(k_{s}\right)$ of snow includes 27 studies since 1886. Sturm et al (1997) concluded by suggesting an empirical model for $k_{s}$ based on snow density, but noted that the large variation in conductivity at a given density was due to the varying microstructure of snow and temperature. Their proposed model predicts lowest values of conductivities among all the reported data so far and primarily chose faceted snow for their experiments, having relatively lower temperature and density than others. Sturm and Johnson (1992) conducted investigation on thermal conductivity of Alaskan depth hoar and found that experimental values were much lower than ever reported. Adams and Sato (1993) hereafter AS-93, used a thermal resistance approach to solve conductance for different constituents and finally demonstrated how important grain contacts $r_{b} / r_{g}$ ratio was in the net- work of ice in determining the value of $k_{s}$. They estimated the dependence of $k_{s}$ on $r_{b} / r_{g}$ as linear. Arons and Colbeck (1995) also reviewed the importance of snow geometry in modelling $k_{s}$ and concluded that characterizing any geometry for this complex material was very difficult.

Review of thermal conductivity data (Yen 1962, 1965, 1981; Schwerdtfeger 1963; Pitman and Zuckerman 1967; Voitkovskiy et al 1975, 1988; Sturm 1991; Sturm and Johnson 1992; Sturm et al 1997) mainly based on field, shows large scatter in data, which can be attributed partly to the density, temperature, microstructure of snow and partly due to the different methods used for the measurements.

Microstructure certainly influences thermal conductivity (Brun et al 1992; AS-93; Arons et al 1994; Arons and Colbeck 1995; Lehning et al 2002; Schneebeli and Sokratov 2004), but exactly how it affects the thermal conductivity is difficult to assess. Measurement of microstructure

Keywords. Thermal conductivity; heat transfer; snow; neck length; snow cover; firn; geophysics; hydrology; modelling; cryosphere. 
using serial sectioning is time consuming, and requires many assumptions to produce microstructural parameters but the new technique known as computed X-ray tomography is least time consuming and free from the assumptions (Schneebeli and Sokratov 2004).

The time dependence of effective thermal conductivity has been shown by Schneebeli and Sokratov (2004) from the laboratory experiments. The results are however, contrary to conventional assumptions. They conducted experiments under temperature gradient and found an increase in the effective thermal conductivity with time for snow of high density. Their finding on low density snow shows that conductivity initially decreases and then increases with time and is quite similar to the study of Sturm and Johnson (1992). It is understood that all the horizontally oriented bonds will disappear due to sublimation process and only those bonds which are in the direction of heat flow will survive and may grow with time (Satyawali 1999). This is the simplest explanation and further consideration should be acknowledged when detailed structural information during metamorphism is known from the X-ray tomography experiments (Schneebeli and Sokratov 2004).

Kaempfer et al (2005) demonstrated that tortuosity factor takes into account the relevant geometry and accounts for the heat transport in the ice matrix. This approach requires threedimensional snow structure. Despite this, it would still be extremely useful to have a conceptual understanding of how microstructure and shape affect the thermal conductivity. AS-93 model envisions a two-dimensional configuration with series and parallel path, building on the model by De Quervain (1973) for heat transfer, but finally presented a one-dimensional model and found it to be satisfactory.

The modern approach to link constitutive parameters is novel to the field of snow cover modelling (Brun et al 1992; Lehning et al 2002). The recent approach has been to analyze thermal conductivity with microstructure (Schneebeli and Sokratov 2004). The importance of microstructural parameters (e.g., grain and bond radius, neck length, grain-spacing and grain shape, etc.) on effective thermal conductivity has been stressed upon by several authors earlier (AS-93; Sturm et al 1997, 2002; Arons and Colbeck 1998).

The aim of the paper is to present a quantitative relationship between thermal conductivity and the microstructure and try to explore the variations in thermal conductivity for a given density range. The hypothesis used is that microstructural variations in grain radius, grain-spacing, and bond radius are sufficient to explain the observed variations of conductivity at a given density. Here, a geometrical model including different shapes (S1, $\mathrm{S} 2$ and S3) influencing thermal conductivity has been proposed. The main reason to choose different shapes is to approximate fine grained snow (high curvature) by spherical grains and faceted snow by cubical grains. In reality, mixed forms are found and therefore cylindrical shape has also been considered, which has both curved and flat surfaces as well.

A steady-state approach has been used to solve the simultaneous heat transfer equations for unit cell (figure 1a). The unit cells comprising the ice grains having spherical, cylindrical and cubical shapes are represented by geometry $\mathrm{S} 1, \mathrm{~S} 2$ and S3 respectively with all the ice grains arranged in a cubic packing. Finally, the model has been extended to snow by assuming a large number of similar unit cells arranged in the direction of heat flow. Computed results for S1 have first been compared with the resistor model of Verma et al (1991) to validate the model. The results obtained have been compared with the empirical model of Sturm et al (1997) and AS-93 model.

\section{Modelling approach}

A simple ball and stick geometry (S1) shown in figure 1(a), has been considered to model the thermal conductivity of an ice/air system in a cubic packing. The grains are connected through bonds having radius $r_{b}$ and neck length $l$. Grain-spacing is set at $h$ and $h^{\prime}$ in vertical and horizontal direction. The vertex of unit cell (figure 1a) rests at the center of all the cylindrical bonds. The assumption of a regular cubic grid is considered in spite of the fact that snow is too varying to treat in a regular manner. Vertical chains or column of grains have been observed during temperature gradient metamorphism (Satyawali 1999; Schneebeli et al 1999) and hence the motivation for this geometry (S1). This geometry however, does not connect the snow vertically as only two grains have been shown bonded in the unit cell separated by a grain-spacing. The geometry excludes the horizontal connection between the snow grains. Since this will confine the purpose of this model especially to snow, nevertheless the present method is able to substantiate the effect of microstructural parameters on the thermal conductivity. In the present work, other geometrical shapes (cylindrical and cubical) as shown in figure 1 (b and $c$ ) have been also considered to address the shape dependency of conductivity apart from the microstructure and temperatures.

The density of unit cell (figure 1a) can be written in terms of grain radius, bond radius, neck length and grain-spacing as: 


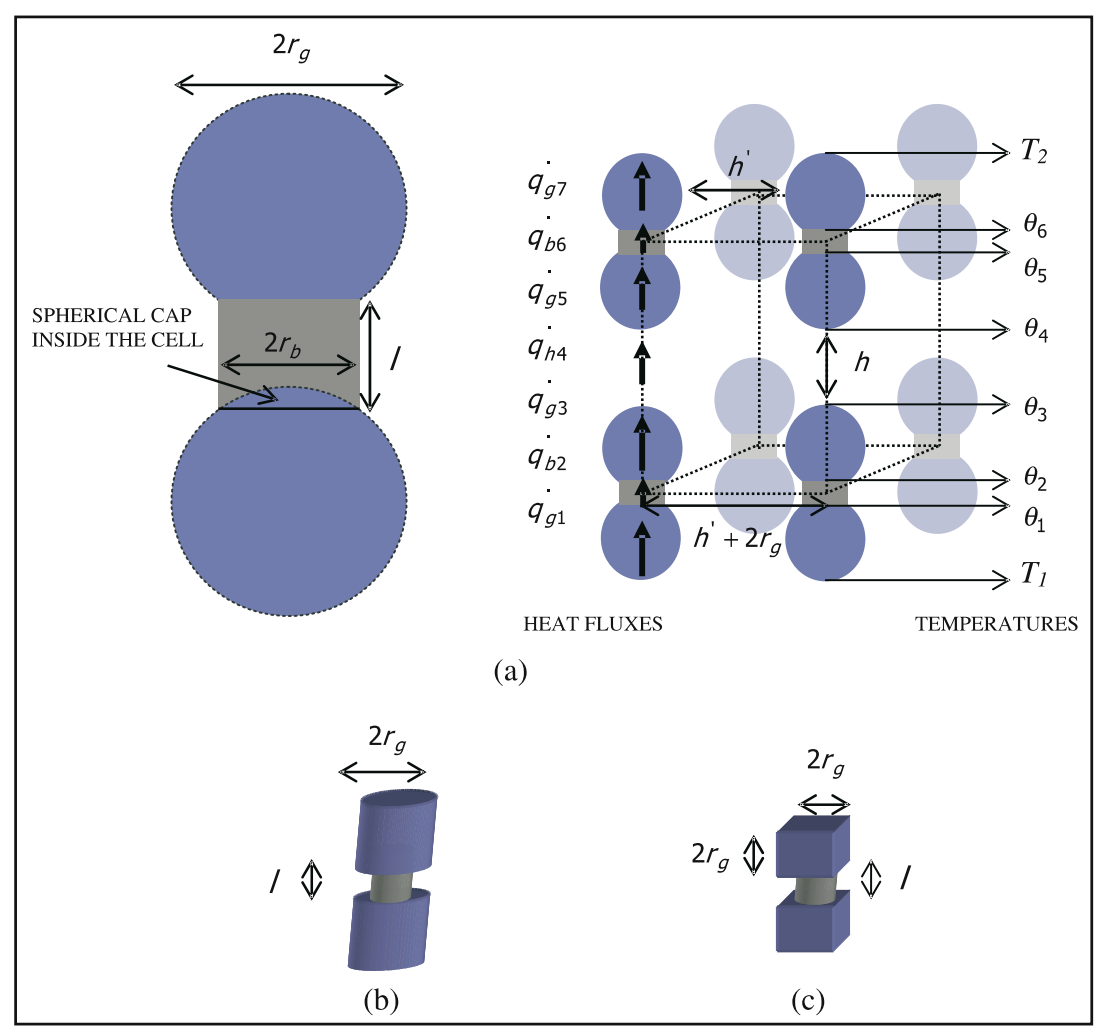

Figure 1. (a) Geometrical model S1 showing arrangement of grains and bonds in a unit cell. Grains and bonds are not highlighted in the second row of unit cell. In the left, one of the similar constituent of unit cell is magnified to show the spherical cap. (b) shows cylindrical shape of grains for geometrical model S2. The spherical grains in (a) are replaced by grains arrangement shown in (b). (c) shows shape of grains for geometrical model S3. Here, spherical grains in (a) are replaced by grains arrangement shown in (c).

$$
\rho_{s 1}=\frac{(8 / 3) \pi r_{g}^{3}+\pi l r_{b}^{2}-(2 / 3) \pi\left(r_{g}-\sqrt{r_{g}^{2}-r_{b}^{2}}\right)^{2}\left(2 r_{g}+\sqrt{r_{g}^{2}-r_{b}^{2}}\right)}{\left(4 r_{g}+h+l-\left(2 r_{g}-2 \sqrt{r_{g}^{2}-r_{b}^{2}}\right)\right)\left(h^{\prime}+2 r_{g}\right)^{2}} \rho_{i} .
$$

The third term in the numerator appeared because volume of spherical cap (figure 1a) is added twice, hence subtracted. In a similar way, the total height of the unit cell is reduced by the height of spherical cap in the denominator. Since volume correction is very small as compared to the first two terms in the numerator (less than $0.5 \%$ for $r_{b} / r_{g}$ ratio less than 0.4 ) and similarly height correction of unit cell is negligible as compared to the first three terms in the denominator (less than $3.9 \%$ for $r_{b} / r_{g}$ ratio less than 0.4$)$. However, with increase in $r_{b} / r_{g}$, these correction terms also increase but in present work, $r_{b} / r_{g}$ is considered to be less than 0.4 and therefore both the correction terms have been neglected. The density of unit cell can be written as:

$$
\rho_{s 1}=\frac{(8 / 3) \pi r_{g}^{3}+\pi l r_{b}^{2}}{\left(4 r_{g}+h+l\right)\left(h^{\prime}+2 r_{g}\right)^{2}} \rho_{i}
$$

\subsection{Governing heat transfer equations for the model (S1)}

To model thermal conductivity, conduction through the ice grains and bonds, conduction through the air and latent heat transfer across the grain-spacing were considered. During the early period of a snow cover buildup, as density is low and temperature gradient is high, vapour diffusion remains dominant mechanism for heat transfer (Colbeck 1993; Arons and Colbeck 1995; Sturm et al 1997). This causes more latent heat to be supplied from warmer to colder region resulting in a higher thermal conductivity of the medium. Since vapour transfer is a function of temperature and temperature gradient across the grain-spacing, it is important to estimate the temperature gradient across the grain-spacing. 


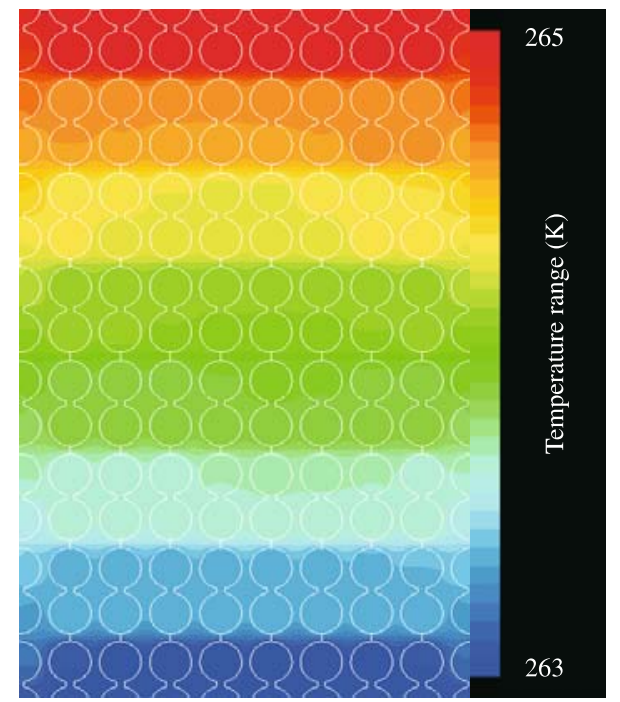

Figure 2. 2-D FE simulation of temperature distribution for unit cell (figure 1a) using Gambit 2.2 and Fluent 6.2. It shows many such cells have been connected vertically to represent a dimension of $26 \mathrm{~mm} \times 40 \mathrm{~mm}$ with lower boundary temperature at $263 \mathrm{~K}$ and upper boundary temperature at $265 \mathrm{~K}$.

The heat fluxes shown in one of the columns in the grains (figure 1a), the necks and the grain-spacing has the same forms as given in Satyawali (1999), except that latent heat has been added into the calculations and heat flow has been considered at the center of grain and bond. It is necessary to obtain first the temperature values at the upper and lower boundaries of a unit cell in the direction of heat flow. To do so, the simultaneous heat conduction equations must first be solved. The heat flux direction has been shown in figure 1(a). It is assumed that temperature is identical in a perpendicular plane of heat flow. This assumption was validated from a $2-\mathrm{D}$ FE simulation of temperature distribution for unit cell S1 (figure 2). However, for natural snow, due to random connections between ice structures, the temperature will vary in the horizontal plane.

Due to symmetry (figure 1a), the following heat transfer equation can be written for the grain situated at the bottom in unit cell:

$\dot{q}_{g 1}=\frac{1}{\left(h^{\prime}+2 r_{g}\right)^{2}}\left[k_{i} A_{g}+k_{a}^{\prime}\left(\left(h^{\prime}+2 r_{g}\right)^{2}-A_{g}\right)\right] \frac{\theta_{0}-\theta_{1}}{2 r_{g}}$.

The first term in the above equation comes from the contribution due to ice part and the second term arises from the air and water vapour medium taken together. It can be calculated that the height of the spherical cap is negligible as compared to the total height of the unit cell and also the neck length and therefore temperature along the height of the spherical cap can be taken as nearly constant. The combined equation for all the four grains along the column in figure 1(a) can be rewritten as:

$$
\begin{aligned}
\dot{q}_{g j}= & \left(\left(k_{i}-k_{a}^{\prime}\right) \frac{A_{g}}{\left(h^{\prime}+2 r_{g}\right)^{2}}+k_{a}^{\prime}\right) \frac{\theta_{j-1}-\theta_{j}}{2 r_{g}}, \\
& j=1,3,5,7 .
\end{aligned}
$$

Here $A_{g}$ is estimated as two-third of $\pi r_{g}^{2}$ for a spherical grain. Since cross-section area of a spherical grain along temperature gradient is not constant, it is averaged for the entire length of the grain. In equation (3), $\theta_{0}=T_{1}$ and $\theta_{7}=T_{2}$ are the reference temperatures, where $T_{1}>T_{2}$.

The heat transfer equation for grain-spacing is:

$$
\dot{q}_{h 4}=k_{a}^{\prime} \frac{\theta_{3}-\theta_{4}}{h} .
$$

Similarly, the combined equation for all the bonds along the column is:

$$
\begin{aligned}
\dot{q}_{b j}= & \left(k_{a}^{\prime}+\frac{A_{b}}{\left(h^{\prime}+2 r_{g}\right)^{2}}\left(k_{i}-k_{a}^{\prime}\right)\right) \frac{\theta_{j-1}-\theta_{j}}{l}, \\
& j=2,6 .
\end{aligned}
$$

The Fourier heat conduction equation for the entire unit cell can be written as:

$$
\dot{q}_{s 1}=k_{s 1} \frac{T_{1}-T_{2}}{8 r_{g}+2 l+h} .
$$

It can be further stated that the height of spherical cap is negligible as compared to the height of the unit cell hence not considered in equation (6). Under steady-state conditions, heat fluxes $\dot{q}_{g 1}, \dot{q}_{b 2}, \dot{q}_{g 3}, \dot{q}_{h 4}, \dot{q}_{g 5}, \dot{q}_{b 6}, \dot{q}_{g 7}$ and $\dot{q}_{s 1}$ will be equal and equations (3) to (5) can be solved as simultaneous equations for $\theta_{1}, \theta_{2}, \theta_{3}, \theta_{4}, \theta_{5}$ and $\theta_{6}$. The values of $\theta_{1}$ and $\theta_{2}$ are obtained as:

$$
\theta_{1}=\frac{T_{1}\left(\frac{2 K}{K_{2}} \frac{l}{2 r_{g}}+\frac{K}{K_{1}} \frac{h}{2 r_{g}}+3\right)+T_{2}}{\frac{2 K}{K_{2}} \frac{l}{2 r_{g}}+\frac{K}{K_{1}} \frac{h}{2 r_{g}}+4} .
$$

and

$$
\theta_{2}=\frac{T_{1}\left(\frac{K}{K_{2}} \frac{l}{2 r_{g}}+\frac{K}{K_{1}} \frac{h}{2 r_{g}}+3\right)+T_{2}\left(\frac{K}{K_{2}} \frac{l}{2 r_{g}}+1\right)}{\frac{2 K}{K_{2}} \frac{l}{2 r_{g}}+\frac{K}{K_{1}} \frac{h}{2 r_{g}}+4}
$$


Here

$$
\begin{aligned}
& K=k_{a}^{\prime}\left(h^{\prime}+2 r_{g}\right)^{2}+\left(k_{i}-k_{a}^{\prime}\right) A_{g}, \\
& K_{1}=k_{a}^{\prime}\left(h^{\prime}+2 r_{g}\right)^{2}
\end{aligned}
$$

and

$$
K_{2}=k_{a}^{\prime}\left(h^{\prime}+2 r_{g}\right)^{2}+\left(k_{i}-k_{a}^{\prime}\right) A_{b} .
$$

Here $K, K_{1}$ and $K_{2}$ are the average effective heat conductivities based on the areal densities taken at the centre of grain, grain-spacing and bonds respectively. The effective conductivity for saturated air $\left(k_{a}^{\prime}\right)$ can be written as (AS-93):

$$
k_{a}^{\prime}=k_{a}+\frac{D P L^{2}}{R^{2} T^{3}} .
$$

Here, the second part of equation (7) comes from the latent heat contribution. From this equation, we can compute $k_{a}^{\prime}$ as $0.03 \mathrm{~W} \mathrm{~m}^{-1} \mathrm{~K}^{-1}$ at $-20^{\circ} \mathrm{C}$, which is higher than the lowest value $\left(0.026 \mathrm{~W} \mathrm{~m}^{-1} \mathrm{~K}^{-1}\right)$ for snow obtained by Sturm and Johnson (1992). It can be interpreted from equation (7) that a change in the diffusion coefficient will change $k_{a}^{\prime}$ and hence the effective thermal conductivity of snow. For simplicity, a fixed value of the diffusion coefficient has been taken in spite of the fact that it varies differently with snow density (Colbeck 1993; Satyawali 2000). The assumption of constant diffusion coefficient is simply because of the lack of sufficient data. By equating equation (6) with any of the equations (3), (4) and (5), $k_{s 1}$ can be obtained as:

$$
k_{s 1}=k_{a}^{\prime} \frac{h+2 l+8 r_{g}}{h+2 l \frac{K_{1}}{K_{2}}+8 r_{g} \frac{K_{1}}{K}} .
$$

From symmetry, $k_{s 1}$ is generalized for $n$ numbers of unit cells as:

$$
k_{s 1}=k_{a}^{\prime} \frac{h+\frac{n+1}{n} l+\frac{n+1}{n} 4 r_{g}}{h+\frac{n+1}{n} l \frac{K_{1}}{K_{2}}+\frac{n+1}{n} 4 r_{g} \frac{K_{1}}{K}},
$$

if $n=1$, we get equation (8), similarly for $n=$ $2,3, \ldots$ and so on. If $n$ is very large, as considered by Yosida (1955) for snow, we can approximate the fraction $(n+1) / n \approx 1$. In this case, equation (9) can be written as:

$$
k_{s 1}=k_{a}^{\prime}\left(\frac{h+l+4 r_{g}}{h+l \frac{K_{1}}{K_{2}}+4 r_{g} \frac{K_{1}}{K}}\right) .
$$

This relation shows the importance of microstructural parameters as overall controlling factor for thermal conductivity. The quantity in brackets is actually the ratio of the microscopic temperature gradient $\left(T G_{\text {pore_s1 }}\right)$ across the grain-spacing to the mean temperature gradient $\left(T G_{\left.\text {mean_s1 }_{-}\right)}\right)$. $T G_{\text {pore_s1 }}$ is computed using equations (4) and (6) as:

$$
\begin{aligned}
T G_{\text {pore } \_s 1} & =\frac{\theta_{4}-\theta_{3}}{h} \\
& =\frac{h+l+4 r_{g}}{h+l \frac{K_{1}}{K_{2}}+4 r_{g} \frac{K_{1}}{K}} T G_{\text {mean } \_s 1} .
\end{aligned}
$$

From equations (10) and (11), $k_{s 1}$ can also be written as:

$$
k_{s 1}=k_{a}^{\prime} \frac{T G_{\text {pore_s } 1}}{T G_{\text {mean_s1 }}},
$$

where $T G_{\text {mean_s1 }}$ and $T G_{\text {pore_s1 }} \neq 0$.

\subsection{Thermal conductivity for cylindrical grains with bonding (S2)}

Geometry S2 is now considered to examine the effect of cylindrical grains on the thermal conductivity. The dimensions of the unit cell are maintained similar to S1. Spherical grains considered in figure 1(a) are replaced by cylindrical grains (figure 1b).

Similar to S1, the effective thermal conductivity for $\mathrm{S} 2$ can be written as:

$$
k_{s 2}=k_{a}^{\prime} \frac{h+l+4 r_{g}}{h+l \frac{K_{1}}{K_{2}}+4 r_{g} \frac{K_{1}}{K}} .
$$

The density of unit cell for S2 can be computed from the following equation:

$$
\rho_{s 2}=\frac{4 \pi r_{g}^{3}+\pi l r_{b}^{2}}{\left(4 r_{g}+h+l\right)\left(h^{\prime}+2 r_{g}\right)^{2}} \rho_{i} .
$$


2.3 Thermal conductivity for cubical grains with bonding (S3)

Another geometry S3 is considered that accounts for the effect of flat geometry on thermal conductivity. The dimensions of the unit cell have not been changed and maintained similar to S1 and S2. Spherical grains considered in figure 1(a) are now replaced by cubical grains of figure 1(c) to conceive geometry S3.

Similar to S1 and S2, thermal conductivity for S3 can be expressed as:

$$
k_{s 3}=k_{a}^{\prime} \frac{h+l+4 r_{g}}{h+l \frac{K_{1}}{K_{3}}+4 r_{g} \frac{K_{1}}{K_{4}}},
$$

where

$$
K_{3}=k_{a}^{\prime}\left(h^{\prime}+2 r_{g}\right)^{2}+\pi r_{b}^{2}\left(k_{i}-k_{a}^{\prime}\right),
$$

and

$$
K_{4}=k_{a}^{\prime}\left(h^{\prime}+2 r_{g}\right)^{2}+4 r_{g}^{2}\left(k_{i}-k_{a}^{\prime}\right) .
$$

Here $K_{3}$ and $K_{4}$ are the average effective heat conductivities based on the areal densities taken at the centre of bond and grain respectively (figure 1c). The density of unit cell for S3 can be written as:

$$
\rho_{s 3}=\frac{16 r_{g}^{3}+\pi l r_{b}^{2}}{\left(4 r_{g}+h+l\right)\left(h^{\prime}+2 r_{g}\right)^{2}} \rho_{i} .
$$

\section{Results and discussion}

The effective thermal conductivity for the idealized geometrical models was obtained for several cases and compared with the existing microstructural based conductivity models (Verma et al 1991; AS-93).

To ensure that physical laws are treated correctly in our approach, results obtained from the S1 model were compared with the model of Verma et al (1991), which is a resistor model based on spherical particles arranged in cubic cell geometry. Their model computes thermal conductivity as:

$$
\begin{gathered}
\lambda_{e}=\frac{\lambda_{f}\left[2.5985 \phi^{1 / 3}\left(\lambda_{s}-\lambda_{f}\right)+3.224 \phi^{-1 / 3} \lambda_{f}\right]}{\left(1-1.2407 \phi^{1 / 3}\right)\left[2.5985 \phi^{1 / 3}\left(\lambda_{s}-\lambda_{f}\right)\right.} . \\
\left.+3.224 \phi^{-1 / 3} \lambda_{f}\right]+\lambda_{f}
\end{gathered}
$$

Figure 3(a) shows the comparison of $\mathrm{S} 1$ (for $r_{b} / r_{g}$ as 0.01 and 0.2 ) with the resistor model of Verma et al (1991). It is found that the conductivity values of S1 and Verma et al (1991) are in close agreement up to density $300 \mathrm{~kg} \mathrm{~m}^{-3}$. The model of Verma et al (1991) gives a higher value of conductivity at higher densities than our model. It can be stated that both models show a similar nonlinear trend and computed values of conductivity are comparable.

Thermal conductivity computed for S1, S2 and S3 using equations (10), (13) and (15) at a temperature of $-5^{\circ} \mathrm{C}$ are shown in figure $3(\mathrm{~b}$ and c) for $r_{b} / r_{g}$ as 0.01 and 0.2 , respectively, along with results obtained from AS-93 and Sturm et al (1997). Grain radius is allowed varying from $0.01 \mathrm{~mm}$ to $0.5 \mathrm{~mm}$ and grain-spacing from $1.0 \mathrm{~mm}$ to $0.01 \mathrm{~mm}$ both horizontally and vertically. Neck length is taken as a fixed value of $0.1 \mathrm{~mm}$. The dependency of thermal conductivity on density is found to be nonlinear as seen in figure 3 (b and c).

Results from the AS-93 model are found to be corresponding with S3 for $r_{b} / r_{g}$ at 0.01 (figure $3 \mathrm{~b}$ ), but for grain contact ratio of 0.2 , results are found in between the values obtained for S1 and S2 (figure 3c). The behaviour of AS-93 model is similar to S2 except that S2 computed lower values of conductivity for higher grain contact ratio. We presume that the grain geometry of AS-93 model is flat for smaller values of $r_{b} / r_{g}$ and curved for larger values of $r_{b} / r_{g}$. The curvature effect comes from the fact that model AS-93 assume spherical grain shape when grains are in contact. It is also concluded from figure 3 (b and c) that curved surfaces contribute higher conductivity than the flat surfaces even for the same density and $r_{b} / r_{g}$. Further it is incorrect to compare S1 with AS-93 model as S1 can only compute conductivity up to $480 \mathrm{~kg} \mathrm{~m}^{-3}$, which has a nonlinear behaviour around this density. Results of AS-93 offer best comparison with S2 (figure 3c) and S3 (figure 3b). While comparing the empirical results of Sturm et al (1997), it is noticed that for lower grain contact ratio the results of Sturm et al (1997) lie between S1 and $\mathrm{S} 2$ (figure $3 \mathrm{~b}$ ) and for higher grain contact ratio the results are close to S2 (figure 3c). This suggests that $\mathrm{S} 2$ is better comparable for both the results of AS-93 and Sturm et al (1997). As the model of Sturm et al (1997) does not give any idea about snow microstructure, it is possible that the comparison might be uncertain due to variability in their experimental datasets.

It is anticipated that increase in ice mass inside a unit cell would result in a higher thermal conductivity as ice is approximately hundred times more conductive than dry air. This can be explained 

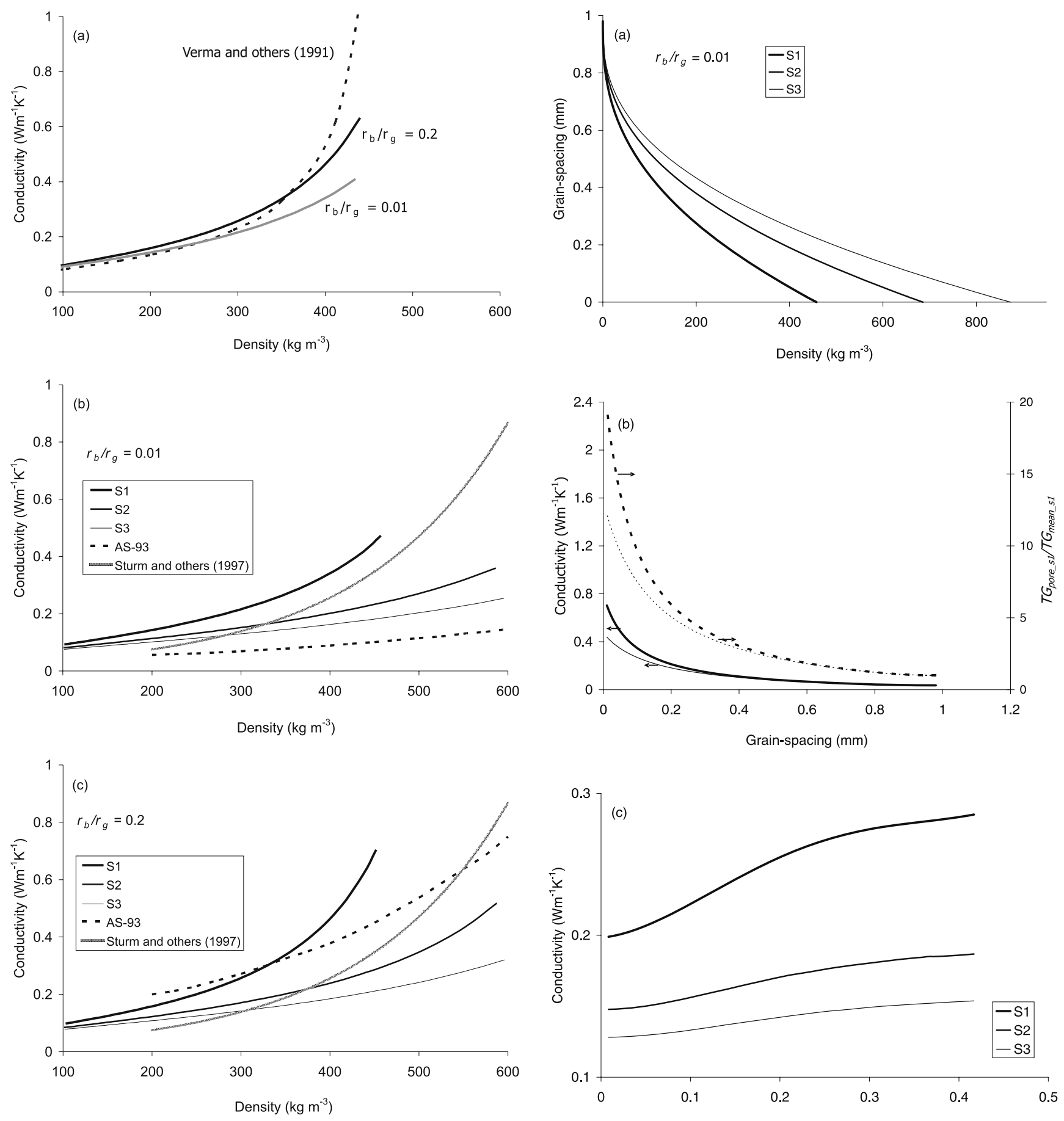

Figure 3. (a) Variation of effective thermal conductivity with density at temperature $-5^{\circ} \mathrm{C}$ for $\mathrm{S} 1$ at different $r_{b} / r_{g}$. Grain size is varied between 0.1 and $0.5 \mathrm{~mm}$, grain-spacing from 0.98 to $0 \mathrm{~mm}$ and neck length is fixed at $0.1 \mathrm{~mm}$. Comparison with Verma et al (1991) is shown. (b) Variation of effective thermal conductivity with density at temperature $-5^{\circ} \mathrm{C}$ for geometrical models (S1, S2 and S3). Grain size is varied between 0.01 and $0.5 \mathrm{~mm}$, grain-spacing from 1.0 to $0.01 \mathrm{~mm}$ and neck length is fixed at $0.1 \mathrm{~mm}$. Comparison with the empirical results of Sturm et al (1997) and AS-93 is shown. (c) Variation of effective thermal conductivity with density at temperature $-5^{\circ} \mathrm{C}$ for geometrical models (S1, S2 and S3). Other parameters are taken similar to (b). Comparison with the empirical results of Sturm et al (1997) and AS-93 is shown.

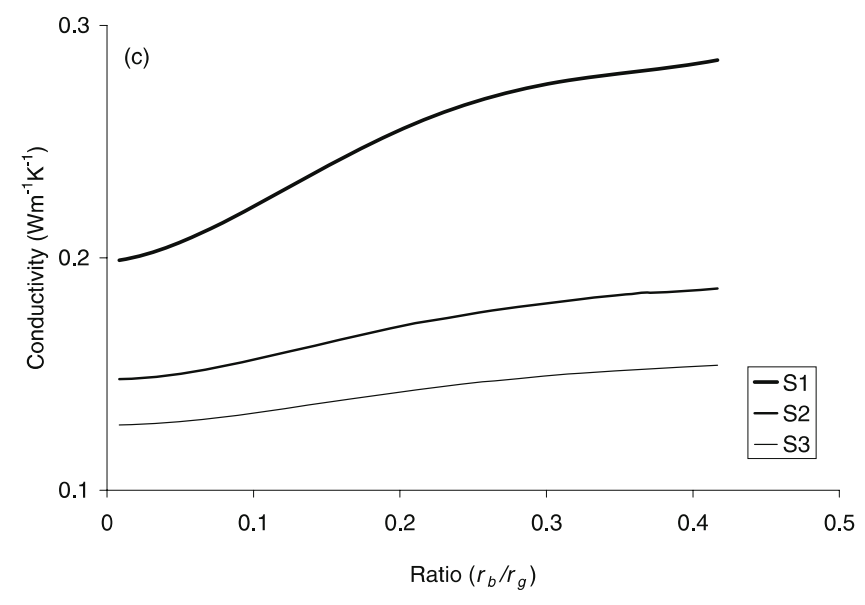

Figure 4. (a) Grain-spacing dependency of the density for models S1, S2 and S3 for $r_{b} / r_{g}$ of 0.01 and a fixed neck length of $0.1 \mathrm{~mm}$. (b) The dependency of effective thermal conductivity and $T G_{\text {pore_s } 1} / T G_{\text {mean_s1 }}$ on grain-spacing is shown for model $\mathrm{S} 1$ at different values of $r_{b} / r_{g}$ and at a constant temperature of $-5^{\circ} \mathrm{C}$. Grain size is allowed to vary between 0.1 and $0.5 \mathrm{~mm}$ and grain-spacing from $0.98 \mathrm{~mm}$ to $0 \mathrm{~mm}$. Neck length is fixed at $0.1 \mathrm{~mm} . r_{b} / r_{g}$ is 0.01 for thin lines (solid and dotted) and 0.2 for thick lines (solid and dotted). (c) Dependence of effective thermal conductivity on $r_{b} / r_{g}$ at a temperature of $-5^{\circ} \mathrm{C}$ and a fixed snow density of $300 \mathrm{~kg} \mathrm{~m}^{-3}$ is shown for $\mathrm{S} 1, \mathrm{~S} 2$ and S3. $r_{g}$ is kept at $0.3 \mathrm{~mm}$ and neck length is fixed as $0.1 \mathrm{~mm}$ for all geometries. 
from figure 4 (a, and b). Figure 4(a) shows that with the increase in density, grain-spacing decreases non-linearly for S1, S2 and S3. Figure 4(b) shows how conductivity changes with the grain-spacing.

We can now determine the dependency of $T G_{\text {pore_s1 }} / T G_{\text {mean_s1 }}$ on grain-spacing from (10) and (12). The dependency is demonstrated in figure 4(b), which shows that the ratio $T G_{\text {pore_s1 }} / T G_{\text {mean_s1 }}$ is non-linearly dependent on grain-spacing. However, this dependency will vary with the changing grain contact ratio. Figure 4(b) also explains that as the grain-spacing becomes smaller (i.e., density increases towards packing density), $T G_{\text {pore_s1 }} / T G_{\text {mean_s1 }}$ becomes larger, which is responsible for higher conductivity value near to packing density. Therefore a strong dependency of $k_{s}$ on snow density can be seen near the packing density.

Once all the grains are in contact, grain-spacing becomes zero and if the neck length is also assumed to be zero, then equations (10), (13) and (15) compute the highest thermal conductivity values, as can be expected when tortuosity is eliminated. In the case of equation (15), the thermal conductivity becomes identical to the conductivity of ice, since $\rho_{s 3}$ becomes $\rho_{i}$ (equation 16 ).

We now give an explanation why grain shape can alter the grain-spacing at constant density and hence affect the thermal conductivity. It is observed from figure 4(a) that for a given density, grain-spacing is lowest for S1 and highest for S3 due to their geometrical shapes. It is concluded from figure $4(\mathrm{~b})$ that $T G_{\text {pore_s } 1} / T G_{\text {mean_s1 }_{1}}$ varies inversely with grain-spacing, we can now generalise that conductivity will be highest for S1 (spherical shapes) and lowest for S3 (flat shapes) from equation (12). The quantification of the shape factor is, however, not included directly in the computation. As equation (12) is applicable for $\mathrm{S} 1$, a general equation for conductivity can be written as:

$$
k_{s}=k_{a}^{\prime} \frac{T G_{\text {pore }}}{T G_{\text {mean }}}
$$

where $T G_{\text {mean }}$ and $T G_{\text {pore }} \neq 0$.

Here $T G_{\text {pore }} / T G_{\text {mean }}$ is the ratio of microscopic temperature gradient to the mean temperature gradient obtained for the models (S1, S2 and S3).

Figure 4(c) demonstrates the variation of thermal conductivity obtained from equations (10), (13) and (15) with a grain contact ratio of $r_{b} / r_{g}$ for a density $300 \mathrm{~kg} \mathrm{~m}^{-3}$ at $-5^{\circ} \mathrm{C}$. These calculations are done for a fixed value of $r_{g}$ at $0.3 \mathrm{~mm}$, neck length of $0.1 \mathrm{~mm}$ and $r_{b}$ varying from 0 to $0.13 \mathrm{~mm}$. To obtain a fixed density for all geometries, grain-
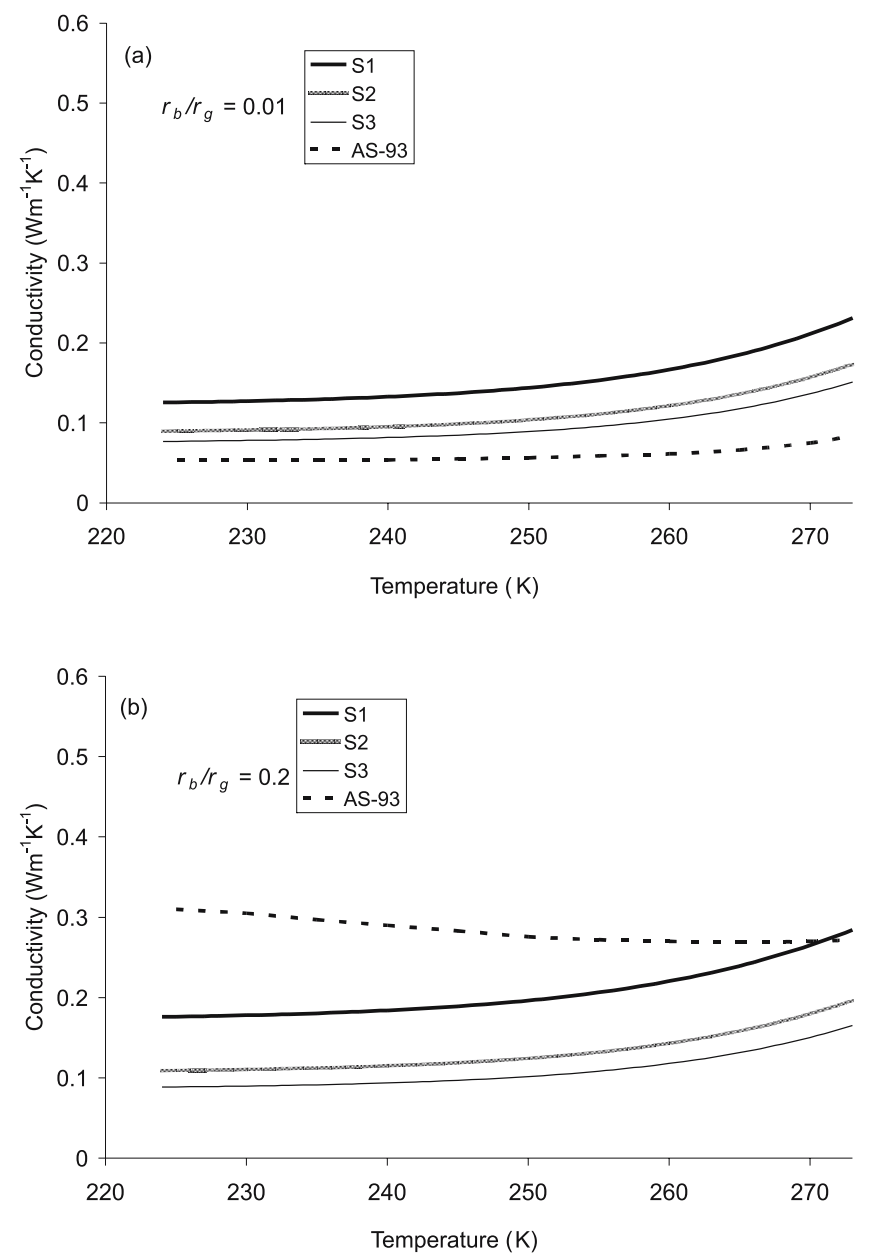

Figure 5. (a) The temperature dependence of the effective thermal conductivity at a density of $300 \mathrm{~kg} \mathrm{~m}^{-3} \cdot r_{g}$ is taken as $0.3 \mathrm{~mm}, l$ as $0.1 \mathrm{~mm}$ for all geometries. $h$ is fixed at $0.102 \mathrm{~mm}$ for $\mathrm{S} 1,0.225 \mathrm{~mm}$ for $\mathrm{S} 2$ and $0.307 \mathrm{~mm}$ for $\mathrm{S} 3$. Comparison with AS-93 is also shown. (b) The temperature dependence of the effective thermal conductivity at a density of $300 \mathrm{~kg} \mathrm{~m}^{-3}$. Values of $r_{g}$ and $l$ are taken similar to (a). Comparison with AS-93 is also shown.

spacing is allowed to vary between $0.10 \mathrm{~mm}$ and $0.11 \mathrm{~mm}$ for $\mathrm{S} 1,0.225$ and $0.229 \mathrm{~mm}$ for $\mathrm{S} 2$ and 0.306 and $0.31 \mathrm{~mm}$ for S3. This way, a constant density of $300 \mathrm{~kg} \mathrm{~m}^{-3}$ is achieved. It is observed that with the increase of the $r_{b} / r_{g}$, conductivity also increases for all the models. However, the increase is more prominent for S1 than for S2 and S3. The thermal conductivity reaches a limiting value as $r_{b} / r_{g}$ becomes larger. The increase in conductivity is found to be nearly $41 \%$ for $\mathrm{S} 1$, $27 \%$ for S2 and $23 \%$ for S3 for the entire range of $r_{b} / r_{g}$ (figure 4c). This increase has not been found in AS-93 model, which shows the linear dependency of conductivity on $r_{b} / r_{g}$, and never reaches a limiting value. Extending the $r_{b} / r_{g}$ linearly to 1, AS-93 computes a high value of conductivity $\left(0.8 \mathrm{~W} \mathrm{~m}^{-1} \mathrm{~K}^{-1}\right)$ for the density $300 \mathrm{~kg} \mathrm{~m}^{-3}$.

Figure 5 ( $\mathrm{a}$ and $\mathrm{b}$ ) shows the variation of the thermal conductivity with temperature for a 
density of $300 \mathrm{~kg} \mathrm{~m}^{-3}$ and $r_{b} / r_{g}$ as 0.01 and 0.2 respectively. The nonlinear increase in conductivity with increasing temperature above $260 \mathrm{~K}$ is due to the fact that saturated water vapour density is more conducting at higher temperature and the dependency of saturated vapour pressure with temperature is exponential. It is assumed that the equilibrium vapour density is governed by the Clapeyron equation (Colbeck 1990).

Figure 5 ( $\mathrm{a}$ and $\mathrm{b}$ ) also compares the results of AS-93 and this is found to be corresponding with S3 for $r_{b} / r_{g}$ at 0.01 . In contrast to this, AS93 model shows a decrease in conductivity with increasing temperature for $r_{b} / r_{g}$ at 0.2 (figure $5 \mathrm{~b}$ ). The conductivity estimated from AS-93 is always higher than the conductivity computed from the present models for the entire range of temperatures. It appears that AS-93 model is based on varying thermal conductivity of ice with temperature, but in the present work a constant value is assumed.

\section{Conclusion}

A geometrical model including different shapes (spherical, cylindrical and cubical) influencing thermal conductivity is proposed to quantify effective thermal conductivity of snow. Steady-state approach is used to solve the simultaneous Fourier equations. A mathematical relation between conductivity and microstructure was established. Results are compared with the resistor model of Verma et al (1991) to ascertain that the physical laws have been treated correctly.

The present work is compared with the empirical model of Sturm et al (1997) and AS-93 model. Model results suggest that S2 is more suitable for the data of Sturm et al (1997). To compare the effect of density, results obtained for S3 are found to be close to the results of AS-93 for $r_{b} / r_{g}$ as 0.01 . For $r_{b} / r_{g}$ as $0.2, \mathrm{~S} 2$ is found comparable with the results of AS-93 for density dependency, while
$\mathrm{S} 1$ is found to be close to AS-93 model for temperature dependency. In addition, present models can explain how geometric shape of snow grains can alter the conductivity. Spherical grain shape model (S1) is found to be estimating higher conductivity values than the cubical grain shape model (S3). Model S1 is restricted up to a snow density of $480 \mathrm{~kg} \mathrm{~m}^{-3}$ and hence has limitation for high density. On the other hand, S3 gives a lower value of conductivity as compared to S1 and S2 but it computes conductivity for a wide range of snow density including ice. All the models (S1, S2 and S3) show dependence of conductivity on $r_{b} / r_{g}$, and this dependence is not as strong as that found in AS-93.

It is assumed that any granular material can be considered either made from perfectly curved surfaces (i.e., S1 in our case) or perfectly flat surface (i.e., S3) particles; any other surface should be a combination of these two geometries. With this assumption, all computed conductivity values from any model should lie between the upper (S1) and lower (S3) values of conductivities. The physical explanation however, for such a wide range of thermal conductivities is lacking from the geometrical models.

Since our modelling approach is analytically based, the advantage of this treatment should be useful for real snow geometries once grain and pore information is available from the X-ray computed tomography (Schneebeli and Sokratov 2004; Kaempfer et al 2005) for the parameterization of complex behaviour (Brun et al 1992; Lehning et al 2002).

\section{Acknowledgements}

Authors would like to thank Dr. R N Sarwade, Director SASE for his permission to publish the work. We also thank Drs. Martin Schneebeli and Matthew Sturm who commented critically on the manuscript which improved the paper significantly. $\mathrm{S}$ K Dewali is thanked for providing FE simulation.

\section{Nomenclature}

\begin{tabular}{lll}
\hline Symbol & Unit & \\
\hline$r_{g}, 2 r_{g}$ & $\mathrm{~m}$ & Grain radius (figures 1a and 1b), grain size (figure 1c) \\
$r_{b}$ & $\mathrm{~m}$ & $\begin{array}{l}\text { Bond radius as shown for figure 1(a) } \\
\text { Grain contact ratio }\end{array}$ \\
$r_{b} / r_{g}$ & & Bond (neck) length as shown for figure 1 \\
$l$ & $\mathrm{~m}$ & Grain-spacing as shown in the figure 1(a)
\end{tabular}




\begin{tabular}{|c|c|c|}
\hline Symbol & Unit & Description \\
\hline$\dot{q}_{g j}, \dot{q}_{h 4}, \dot{q}_{b j}$ & $\mathrm{~W} \mathrm{~m}^{-2}$ & $\begin{array}{l}\text { Heat flow rates at the center of the ice grains, between grain-spacing } \\
\text { and at the center of the ice bonds respectively. Subscripts } g, h \text {, and } \\
b \text { are used to denote grain, grain-spacing and bond, and } j \text { is used } \\
\text { to denote the element number from the bottom (figure 1a). }\end{array}$ \\
\hline$\dot{q}_{s 1}$ & $\mathrm{~W} \mathrm{~m} \mathrm{~m}^{-2}$ & Bulk heat transfer for unit cell (figure 1a) over an area of $\left(h^{\prime}+2 r_{g}\right)^{2}$ \\
\hline$k_{s}$ & $\mathrm{~W} \mathrm{~m}^{-1} \mathrm{~K}^{-1}$ & Effective thermal conductivity of snow \\
\hline$k_{s 1}, k_{s 2}, k_{s 3}$ & $\mathrm{~W} \mathrm{~m} \mathrm{~m}^{-1} \mathrm{~K}^{-1}$ & $\begin{array}{l}\text { Thermal conductivity for geometries } \mathrm{S} 1, \mathrm{~S} 2 \text { and } \mathrm{S} 3 \text { respectively } \\
\text { (figure 1) }\end{array}$ \\
\hline$k_{i}$ & $\mathrm{~W} \mathrm{~m}^{-1} \mathrm{~K}^{-1}$ & Conductivity of ice $\left(2.2 \mathrm{~W} \mathrm{~m}^{-1} \mathrm{~K}^{-1}\right)$ \\
\hline$k_{a}, k_{a}^{\prime}$ & $\mathrm{W} \mathrm{m}^{-1} \mathrm{~K}^{-1}$ & Conductivity of dry air and saturated air respectively \\
\hline$K, K_{1}, K_{2}$ & & $\begin{array}{l}\text { Average effective heat conductivities based on the areal densities } \\
\text { taken at the centre of grain, grain-spacing and bond respectively } \\
\text { (figure 1a) }\end{array}$ \\
\hline$K_{3}, K_{4}$ & & $\begin{array}{l}\text { Average effective heat conductivities based on the areal densities } \\
\text { taken at the centre of bond and grain respectively (figure 1c) }\end{array}$ \\
\hline$A_{a v}$ & $\mathrm{~m}^{2}$ & $=\left(h^{\prime}+2 r_{g}\right)^{2}$, cross section area of unit cell (figure 1a) \\
\hline$A_{g}, A_{b}$ & $\mathrm{~m}^{2}$ & $\begin{array}{l}\text { Average cross-section area of grain and bond respectively, perpen- } \\
\text { dicular to the direction of heat flow. } A_{g} \text { is } \pi r_{g}^{2} \text { for cylindrical grains, } \\
2 / 3 \pi r_{g}^{2} \text { for spherical grains and } 4 r_{g}^{2} \text { for cubical grains }\end{array}$ \\
\hline$T_{1}, T_{2}$ & $\mathrm{~K}$ & Reference temperatures $\left(T_{1}>T_{2}\right)$ for unit cell (figure 1a) \\
\hline$T$ & $\mathrm{~K}$ & Average temperature of unit cell (figure 1a) \\
\hline$\theta_{0}$ & $\mathrm{~K}$ & $=T_{1}$, bottom reference temperature of unit cell \\
\hline$\theta_{1}, \theta_{2}, \ldots, \theta_{6}$ & $\mathrm{~K}$ & Temperatures along the heat flow direction (figure 1a) \\
\hline$T G_{\text {mean_s1 }}$ & $\mathrm{K} \mathrm{m}^{-1}$ & Average temperature gradient for unit cell in figure 1(a) \\
\hline$T G_{\text {pore_s } 1}$ & $\mathrm{~K} \mathrm{~m}^{-1}$ & Microscopic temperature gradient at grain-spacing (figure 1a) \\
\hline$\rho_{s 1}, \rho_{s 2}, \rho_{s 3}$ & $\mathrm{~kg} \mathrm{~m}{ }^{-3}$ & Density of unit cell for S1, S2 and S3 \\
\hline$\rho_{i}, \rho_{s}$ & $\mathrm{~kg} \mathrm{~m}{ }^{-3}$ & Density of ice and snow respectively \\
\hline$D$ & $\mathrm{~m}^{2} \mathrm{~s}^{-1}$ & Diffusion coefficient of water vapour in air, $2.02 \times 10^{-5} \mathrm{~m}^{2} \mathrm{~s}^{-1}$ \\
\hline$T_{0}$ & $\mathrm{~K}$ & Reference temperature $(273 \mathrm{~K})$ \\
\hline$P$ & $\mathrm{~N} \mathrm{~m}^{-2}$ & Saturation water vapour pressure over an ice surface \\
\hline$P_{0}$ & $\mathrm{~N} \mathrm{~m}^{-2}$ & Saturation water vapour pressure over an ice surface at triple point \\
\hline$L$ & $\mathrm{~J} \mathrm{~kg}^{-1}$ & Latent heat of sublimation, $2.838 \times 10^{6} \mathrm{~J} \mathrm{~kg}^{-1}$ \\
\hline$R$ & $\mathrm{~J} \mathrm{~kg}^{-1} \mathrm{~K}^{-1}$ & Universal gas constant, $462 \mathrm{~J} \mathrm{~kg}^{-1} \mathrm{~K}^{-1}$ \\
\hline$\lambda_{e}$ & $\mathrm{~W} \mathrm{~m} \mathrm{~m}^{-1} \mathrm{~K}^{-1}$ & Effective thermal conductivity using resistor approach \\
\hline$\lambda_{f}$ & $\mathrm{~W} \mathrm{~m}^{-1} \mathrm{~K}^{-1}$ & Medium thermal conductivity \\
\hline$\lambda_{s}$ & $\mathrm{~W} \mathrm{~m}^{-1} \mathrm{~K}^{-1}$ & Thermal conductivity of the solid inclusion \\
\hline$\phi$ & & Volume fraction of solid (ice) \\
\hline
\end{tabular}




\section{References}

AS-93 (Adams E E and Sato A 1993) Model for effective thermal conductivity of a dry snow cover composed of uniform ice spheres; Annl. Glaciol. 18 300-304.

Arons E M, Colbeck S C, McGilvary W R and Petrenko V F 1994 Effective medium approximation for snow thermal conductivity; In: Proceedings of the International Symposium on Snow and Related Manifestations 'SNOWSYMP 94', Manali, 26-28 Sep 1994, Agrawal K C (ed) Snow and Avalanche Study Establishment 141-145.

Arons E M and Colbeck S C 1995 Geometry of heat and mass transfer in dry snow: A review of theory and experiment; Review of Geophysics 33(4) 463-493.

Arons E M and Colbeck S C 1998 Effective medium approximation of sensible heat in dry snow; Int. J. Heat Mass Transfer 41 2653-2666.

Brun E, David P, Sudul M and Brunot G 1992 A numerical model to simulate snow-cover stratigraphy for operational avalanche forecasting; J. Glaciol. 38(128) $13-22$.

Colbeck S C 1990 Vapor-pressure dependence on temperature in models of snow metamorphism; J. Glaciol. 36(124) 351-353.

Colbeck S C 1993 The vapor diffusion coefficient for snow; Water Resour. Res. 29(1) 109-115.

De Quervain M R 1973 Snow structure, heat and mass flux through snow; In: The Role of Snow and Ice in Hydrology; Banff, Canada, September 1972, International Association of Hydrological Sciences: Wallingford; 203-226.

Kaempfer T U, Schneebeli M and Sokratov S A 2005 A microstructural approach to model heat transfer in snow; Geophys. Res. Lett. 32 L21503 doi:10.1029/ 2005GL023873.

Lehning M, Bartelt P, Brown R L, Fierz C and Satyawali P K 2002 A physical SNOW PACK model for the Swiss avalanche warning - part II: snow microstructure; Cold Regions Sciences and Technology 35(3) 147-167.

Satyawali P K 1999 Bond growth under temperature gradient; Def. Sc. Journal 49(5) 363-370.

Satyawali P K 2000 Diffusivity and vapour flow into snow during phase change; Ann. Glaciol. 31 445-450.

Schneebeli M, Pielmeier C and Johnson J B 1999 Measuring snow microstructure and hardness using a high resolution penetrometer; Cold Regions Sciences and Technology 30(1-3) 101-114.

Schneebeli M and Sokratov S A 2004 Tomography of temperature gradient metamorphism of snow and associated changes in heat conductivity; Hydrol. Processes 18(18) 3655-3665, doi:10.1002/hyp.5800.

Schwerdtfeger P 1963 Theoretical derivation of the thermal conductivity and diffusivity of snow; Int. Assoc. Sci. Hydrol. Publ. 61 75-81.

Sturm M 1991 The role of thermal convection in heat and mass transport in the subarctic snow cover; CRREL Rep. 91-19.

Sturm M and Johnson J B 1992 Thermal conductivity measurement of depth hoar; J. Geophys. Res. 97(B2) $2129-2139$.

Sturm M, Holmgren J, König M and Morris K 1997 The thermal conductivity of seasonal snow; J. Glaciol. 43(143) 26-41.

Sturm M, Perovich D K and Holmgren J 2002 Thermal conductivity and heat transfer through the snow on the ice of the Beaufort Sea; J. Geophys. Res. 107 X-1 doi:10.1029/2000JC000409.

Verma L S, Shrotriya A K, Singh R and Chaudhry D R 1991 Thermal conduction in two phase materials with spherical and non-spherical inclusions; J. Phys. D: Appl. Phys. 24 1729-1737.

Voitkovskiy K F, Golubev V N, Lapteva N I, Troshkina Ye S, Ushakova L A and Pavlov A V 1975 Mass transfer and metamorphism in snow cover; International Association of Hydrological Sciences Publication 114 (Symposium at Grindelwald 1974-Snow Mechanics) 16-24.

Voitkovskii K F, Golubev V N, Sazonov A V and Sokratov S A 1988 New data on the water vapour diffusion coefficient in snow; Mater. Gliatsiologicheskikh Issled. 63 76-81.

Yen Y C 1962 Eeffective thermal conductivity of ventilated snow; J. Geophys. Res. 67(3) 1091-1098.

Yen Y C 1965 Effective thermal conductivity and water vapour diffusivity of naturally compacted snow; J. Geophys. Res. 70(8) 1821-1825.

Yen Y C 1981 Review of thermal properties of snow, ice and sea ice; CRREL Rep. 81-10.

Yosida G 1955 Physical studies on deposited snow. I. Thermal properties; Contrib. Inst. Low Temp. Sci. Ser. A 7 19-74. 